\title{
Intersubband Absorption in Quantum Dash Nanostructures
}

\author{
J.V. CRnjanski* and D.M. Gvozdić \\ School of Electrical Engineering, University of Belgrade, Serbia
}

\begin{abstract}
The band structure and corresponding intersubband absorption coefficient of quantum dash structures are modeled using a self-consistent single-band effective-mass approach and dipole approximation. Size fluctuation of the quantum dash ensemble, described with Gaussian distribution, is included in the model and its effects on the absorption spectrum are analyzed. The profile of the absorption spectrum is suitable for fitting with a sum of Gaussian functions. We find that the quantum dash ensemble has a pronounced and broad absorption spectrum in mid-infrared region, which becomes broader and more asymmetric with increase of the size fluctuation. In addition, for very large size fluctuation, the spectrum becomes flattened in relatively wide wavelength range.
\end{abstract}

PACS numbers: 73.21.Hb, 78.67.Lt, 78.67.Uh

\section{Introduction}

Self-assembled quantum nanostructures have already attracted considerable interest for application in photonics and optical communications, due to their new and advanced electronic and optical properties and their self-assembling process of growth, which does not require any additional lithographic steps. The most interesting applications of these structures are usually related to semiconductor lasers and optical amplifiers [1], where self-assembled quantum dots (QDs) are used as an active region, providing for low threshold current [2], low chirp and small line width enhancement factor [3]. However, studies of QDs lasers are limited on GaAs substrates with emission wavelength smaller than $1.3 \mu \mathrm{m}$. The growth of self-assembled nanostructures on InP substrates led to the discovery of quantum dash structures, with emission wavelength around $1.55 \mu \mathrm{m}$, which have high potential for applications in optical communications [4].

Quantum dashes (QDH) are wirelike semiconductor nanostructures, which self-assemble during the growth process. One of the prominent features of QDHs is significant size fluctuation in respect of widths, heights, and lengths. This feature results in broadening of sharp peaks in the density of states, as usually found in ideal quantum wires, and broadening of gain spectrum, when QDHs are used as an active medium of lasers $[4,5]$.

However, the application of QDHs so far has been related to interband optical transitions, which occur among the subbands of the conduction and the valence band. To the best of our knowledge, the intersubband transitions in QDHs have not attracted attention at all, although they were considered in the case of QDs [6]. Thus, in our work, we investigate intersubband transitions in the

\footnotetext{
* corresponding author; e-mail: jasna.crnjanski@etf.rs
}

conduction band of quantum dashes, taking into account fluctuation of their size, described by a Gaussian distribution [5]. The aim of this work is exploration of QDH optical properties for application in mid-infrared light detection.

In the next section, we present a theoretical approach implemented in our calculation. In Sect. 3, we discuss the calculated intersubband absorption coefficient for ensembles of QDHs with different size fluctuations. In the final section, we formulate our conclusions.

\section{Theoretical analysis}

In order to determine optical intersubband properties, we first calculate intersubband absorption coefficient of a single $\mathrm{QDH}$ for various widths and heights at room temperature $(T=300 \mathrm{~K})$. Then, we carry out ensemble average by summation of the previously calculated absorption coefficients, weighted by the probability of QDH dimensions. The electronic band structure calculation is performed self-consistently in the parabolic approximation, as in [7]. Although nonparabolicity may affect intersubband absorption spectra [7], we focus on the effect of the Hartree potential, which is more prominent.

In calculating the intersubband absorption coefficient of a single QDH, we consider a single modulation-doped quantum dash based on InAs well material, which is grown on and surrounded by GaAs. Material parameters for InAs and GaAs, as well as the conduction band offset, are taken from [8]. The cross-section profile of the QDH is approximated with an elongated semi-cylindrical geometry [9] as presented in Fig. 1. Since quantum dash is an elongated InAs island [10], the QDH well material has almost a quantum-wire nature. Thus, the quantization in the longitudinal QDH direction (z-direction) leads to a quasi-continuous subband structure, whose energy is well approximated with parabolic dependence on correspond- 
ing wave vector $\left(k_{z}\right)$. Therefore, in our analysis we approximate the QDHs with equally long wires and concentrate only on the carrier confinement in the transversal direction. We assume that electrons necessary for optical transitions are provided from two doped layers (with donor concentration $N_{\mathrm{dD}}=5 \times 10^{18} \mathrm{~cm}^{-3}$ ), which are separated from QDH by spacer layer (with donor concentration $N_{\mathrm{dS}}=10^{15} \mathrm{~cm}^{-3}$ ), as shown in Fig. 1. The spacer layer width is used to control the carrier concentration in the dash. The dimensions of the QDH in transversal plane are width $(W)$ and height $(H)$.

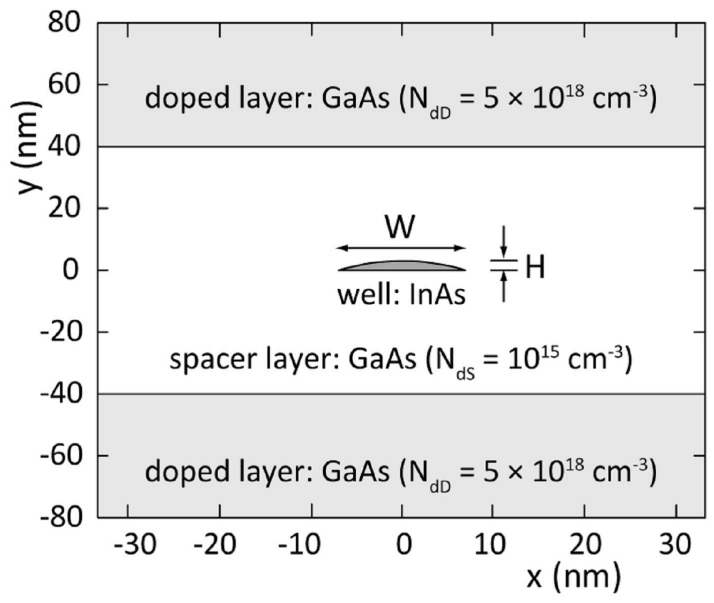

Fig. 1. The QDH profile and domain of the self-consistent solving.

Starting from the band structure, the intersubband absorption coefficient of a single QDH is calculated in the dipole approximation for two directions of light polarization ( $X$ and $y$, as indicated in Fig. 1), similarly as in [7]:

$$
\begin{aligned}
& \alpha(\omega)=\frac{2 q^{2}}{\tilde{n} \varepsilon_{0} m_{0}^{2} c \omega S} \sum_{i, f} \int_{0}^{\infty}\left|\Pi_{i f}^{x y}\right|^{2}\left[f\left(E_{i}\right)-f\left(E_{f}\right)\right] \\
& \quad \times L\left(\hbar \omega, \hbar \omega_{i f}\right) \mathrm{d} k_{z} .
\end{aligned}
$$

In Eq. (1), $E_{j}$ is the energy of the initial $(j=i)$ and the final $(j=f)$ bound state for a given value of the wave vector $k_{z}$ in the longitudinal direction of the dash. With $\hbar \omega_{i f}=E_{i}-E_{f}$ we denote photon energy, $f(E)$ is the Fermi-Dirac function, $\Pi_{i f}$ is the momentum matrix element, $q$ the electronic charge, $\tilde{n}$ the group refractive index, $c$ the velocity of light, $\omega$ the photon frequency, $S$ the cross-sectional area of the QDH, and $\alpha(\omega)$ is absorption coefficient dependent on the structure cross-section. $L\left(\hbar \omega, \hbar \omega_{i f}\right)$ is the Lorentzian that takes line broadening into account. In our calculation we assume the broadening parameter $\Gamma=7 \mathrm{meV}$ [10].

Quantum dashes are grown by self-assembled epitaxial growth, which gives a wide stochastic size distribution, leading to an energy spreading of the confined subbands, each contributing to the broadening of the absorption spectrum. The dash size fluctuation is modeled assuming that each QDH dimension fluctuates with an independent Gaussian distribution with average value $W_{0}$,
$H_{0}$ and standard deviation $\sigma_{W}, \sigma_{H}$ respectively. In our calculation, we assume that the average width and height of QDHs are $W_{0}=14 \mathrm{~nm}$ and $H_{0}=3 \mathrm{~nm}$, respectively. According to [5], the size fluctuation of the QDH dimensions varies between 10 and $30 \%$ of the average dimensions. The probability $\mathrm{d} P(W, H)$ of finding a $\mathrm{QDH}$ with dimension in the ranges $(W, W+\mathrm{d} W)$ and $(H, H+\mathrm{d} H)$ is given by

$$
\begin{aligned}
& \mathrm{d} P(W, H)=\frac{1}{2 \pi \sigma_{W} \sigma_{H}} \\
& \quad \times \exp \left(-\frac{\left(W-W_{0}\right)^{2}}{2 \sigma_{W}^{2}}-\frac{\left(H-H_{0}\right)^{2}}{2 \sigma_{H}^{2}}\right) \mathrm{d} W \mathrm{~d} H .
\end{aligned}
$$

The size fluctuation is characterized by parameter $\Delta$ which is defined as $\Delta=\sigma_{W} / W_{0}=\sigma_{H} / H_{0}$, as we assume identical fluctuations along the $x$ and $y$ directions. For $\Delta=15 \%$ the size distribution of the QDH ensemble is given in Fig. 2.

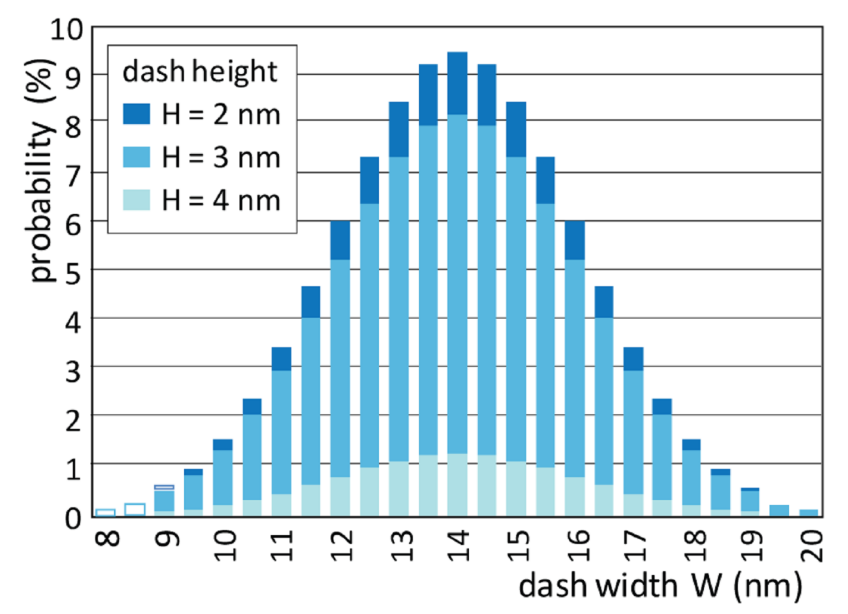

Fig. 2. The size distribution of the QDH ensemble for size fluctuation $\Delta=15 \%$.

\section{Results and discussion}

The intersubband absorption coefficient for size fluctuation $\Delta=15 \%$ of QDH ensemble is shown in Fig. 3 . This size fluctuation corresponds to the QDH ensemble, given in Fig. 2, for which the QDH width varies from 8 to $20 \mathrm{~nm}$, while the height changes from $2 \mathrm{~nm}$ to $4 \mathrm{~nm}$, with discretization steps $\delta_{H}=1 \mathrm{~nm}$ and $\delta_{W}=0.5 \mathrm{~nm}$, respectively. The absorption spectrum is mainly determined by transitions from the ground to the first excited state, except for the subset of the QDH ensemble with dimensions larger than the average. This subset allows the existence of the second or even third excited state in the QDHs and corresponding intersubband transitions. For QDHs with dimensions $(W, H)=\{(8 \mathrm{~nm}, 3 \mathrm{~nm})$, $(8.5 \mathrm{~nm}, 3 \mathrm{~nm}),(9 \mathrm{~nm}, 2 \mathrm{~nm})\}$ represented with white bars in Fig. 2, only the ground state exists, causing no effect on the absorption.

Figure 3 shows that the absorption coefficient for the polarization in the $x$-direction consists of densely dis- 
wavelength $(\mu \mathrm{m})$

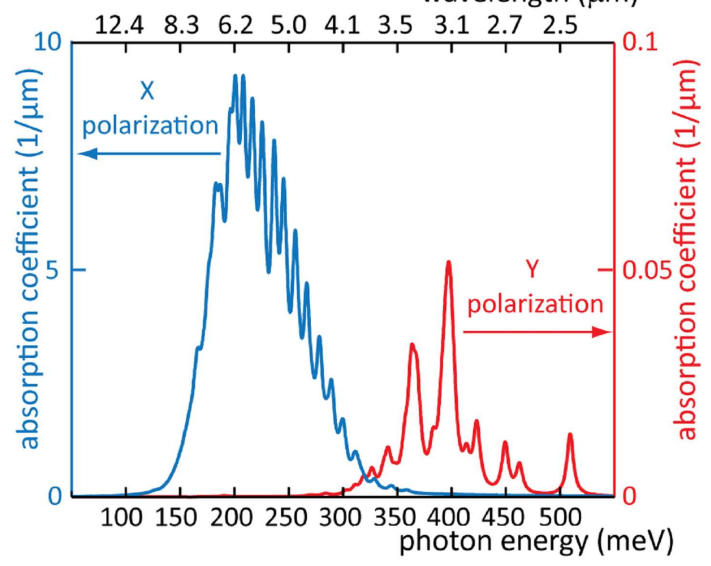

Fig. 3. The absorption coefficient for size fluctuation $\Delta=15 \%$ of the QDH ensemble for the $X$-polarization (left axis) and the $Y$-polarization (right axis).

tributed absorption peaks, originating from the individual QDHs in the ensemble. The superposition of the peaks leads to a broad absorption in the wavelength range between $3.5 \mu \mathrm{m}$ and $10 \mu \mathrm{m}$, with the maximum absorption at $6.19 \mu \mathrm{m}$. Due to relatively small heights of the QDH ensemble, the shape of the wave functions for all confined states is such that maximums of the wave functions modulo extend only in the $x$-direction. Therefore, the transitions from the ground to the first excited state have strong matrix elements for $X$-polarization and almost negligible matrix elements for the $Y$-polarization. However, the existence of higher excited states for the subset of the QDH ensemble with larger dimensions, allows the transitions with relatively small matrix elements in the $y$-direction for higher photon energies. The $Y$-polarization absorption coefficient is approximately 200 times weaker than absorption coefficient for $X$-polarization and consists of several isolated absorption peaks in the wavelength range between $2.3 \mu \mathrm{m}$ and $4.1 \mu \mathrm{m}$. The major reason for the relatively large contribution of these transitions, in spite of small matrix elements, is a rather large difference in subband population.

A further reduction of the discretization steps leads to a much smoother absorption spectrum for the $X$-polarization, for which the values of the adjacent peaks and dips approximately converge to their average values. Thus, we find that discretization beyond a certain level can be successfully replaced by the data fit of the calculated QDH ensemble absorption.

In order to investigate the influence of the size fluctuation on the absorption spectrum, we calculate the absorption coefficient for $\Delta=5,15$ and $30 \%$. In Fig. 4, dotted lines represent the calculated absorption coefficient for all three cases of size fluctuation, while solid lines correspond to data fits of the calculated absorption coefficient. The fitting function, chosen as a sum of Gaus- sians, enables us to predict the absorption spectrum for infinitely small discretization steps. The fitting function and corresponding number of terms in the sum $(n)$ for each size fluctuation is given in Fig. 4. Due to relatively small size fluctuation of the QDH ensemble for $\Delta=5 \%$, the absorption spectrum, although wider, resembles the form of the absorption spectrum for a quantum wire [7]. Although the shape of this spectrum is relatively simple, it requires $n=3$ terms in the fitting sum. The reason for that are extremely sharp peaks caused by small number of QDHs in the ensemble.

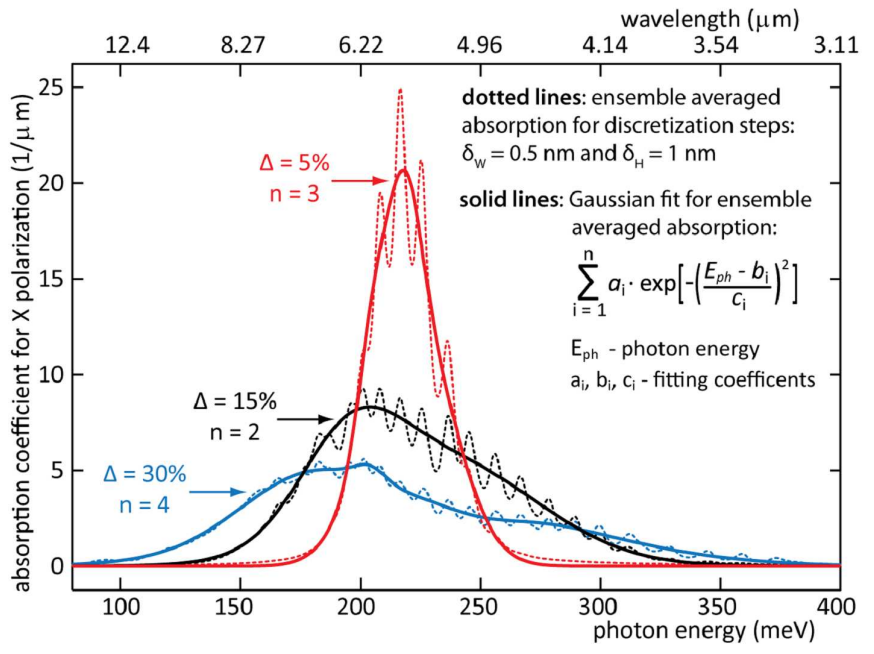

Fig. 4. The calculated absorption coefficient (dotted lines) for size fluctuations $\Delta=5 \%, 15 \%$ and $30 \%$. Solid lines represent corresponding data fits.

The increase of the size fluctuation, leads to a broadening of the absorption spectrum. In addition to that, for increased size fluctuation contribution to the spectrum is significantly different for larger and smaller heights of QDHs in the ensemble. In this case, the energy separation between subbands in QDHs increases more abruptly with reduction of the QDH dimensions for smaller QDHs. This leads to a much wider energy range of allowed transitions for smaller than for larger QDHs. As the smaller QDHs determine the high-energy part of the spectrum, while larger are assigned to the low-energy part, an increase of the size fluctuation leads to a more asymmetric shape of the spectrum, which is simultaneously shifted towards lower photon energies. Thus, the absorption spectrum for $\Delta=15 \%$ is much wider and more asymmetric than for $\Delta=5 \%$. The same is valid for the spectrum for $\Delta=30 \%$ in comparison with $\Delta=15 \%$. Moreover, for $\Delta=30 \%$, the absorption spectrum is considerably flattened in wavelength range from $6.2 \mu \mathrm{m}$ to $7.5 \mu \mathrm{m}$.

\section{Conclusions}

In this paper, we present and analyze the calculation of the intersubband absorption coefficient of QDH nanostructures. We show that statistical nature of the dash 
size leads to broad absorption spectra that can be well approximated with a sum of Gaussian functions. For size fluctuations, QDH profile and material system considered in this work, the fitting sum comprises relatively small number of terms (up to 4). The absorption spectrum lies in mid-infrared wavelength range from $3.5 \mu \mathrm{m}$ to $12 \mu \mathrm{m}$. It is also observed that the absorption spectra will become broader, more asymmetric and flattened, with an increase of the size fluctuation. Such broad and flattened absorption spectrum is suitable for detection or modulation of mid-infrared radiation, terabit optical communications or ultraprecision metrology and spectroscopy [11].

\section{Acknowledgments}

This work is supported by the Serbian Ministry of Science under project no. 160001.

\section{References}

[1] P. Bhattacharya, Z. Mi, Proc. IEEE 95, 1723 (2007).
[2] A. Stintz, G.T Liu, H. Li, L.F. Lester, K.J. Malloy, IEEE Photon. Technol. Lett. 12, 591 (2000).

[3] H. Saito, K. Nishi, A. Kamei, S. Suguo, IEEE Photon. Technol. Lett. 12, 1298 (2000).

[4] J.P. Reithmaier, G. Eisenstein, A. Forchel, Proc. IEEE 95, 1779 (2007).

[5] J.H. Wei, K.S. Chan, J. Appl. Phys. 97, 123524 (2005).

[6] P. Bhattacharya, X. Su, G. Ariyawansa, A.G.U. Perera, Proc. IEEE 95, 1828 (2007).

[7] J. Crnjanski, D. Gvozdić, J. Appl. Phys. 101, 013104 (2007).

[8] I. Vurgaftman, J.R. Meyer, L.R. Ram-Mohan, J. Appl. Phys. 89, 5815 (2001).

[9] M. Gioannini, IEEE J. Quantum Electron. 40, 364 (2004).

[10] H. Dery, E. Benisty, A. Epstein, R. Alizon, V. Mikhelashvili, G. Eisenstein, R. Schwertberger, D. Gold, J.P. Reithmaier, A. Forchel, J. Appl. Phys. 95, 6103 (2004).

[11] C. Gmachl, D.L. Sivco, R. Colombelli, F. Capasso, A.Y. Cho, Nature 415, 883 (2002). 